\title{
Mapping Magnetic Properties of Materials At Atomic Spatial Resolution
}

\author{
Jan Rusz ${ }^{1}$, Juan-Carlos Idrobo ${ }^{2}$, Alexander Edström ${ }^{1}$, Jakob Spiegelberg ${ }^{1}$, and Somnath Bhowmick ${ }^{3}$ \\ 1. Department of Physics and Astronomy, Uppsala University, Uppsala, Sweden \\ 2. Center for Nanophase Materials Sciences, Oak Ridge National Laboratory, Oak Ridge, TN, USA \\ 3. Indian Institute of Technology, Kanpur, India
}

Quantitative characterization of magnetic materials at atomic resolution is one of important challenges for the aberration-corrected scanning transmission electron microscopy (STEM). The ability to measure spin and orbital magnetic moment with atomic spatial resolution at surfaces, across interfaces, or nearby defects, would provide unprecedented new insights into the magnetism at the shortest scale. A candidate experimental technique to achieve this is called electron magnetic circular dichroism (EMCD; [1]). EMCD is analogous to the well established x-ray magnetic circular dichroism, which allows to quantify spin and orbital magnetic moments from spectra measured at core level edges of the magnetic elements.

In recent years, several research groups worldwide are investing substantial effort into high spatial resolution EMCD. This has been largely motivated by the introduction of electron vortex beams (EVBs) $[2,3,4]$ into the world of transmission electron microscopy (TEM). It has been suggested [3] that EVBs will allow measurement of EMCD directly at the transmitted beam. If confirmed, this would bring an enormous improvement of signal to noise ratio compared to the classical EMCD, where spectra are acquired in between the Bragg spots, using electron beams with small convergence angles [5].

However, it was shown recently [6], that to acquire EMCD at the transmitted beam requires that the size of the EVB must be small enough to allow for atomic resolution. Expressed in terms of convergence angles and resulting convergent beam electron diffraction (CBED) pattern, the convergence angle must be large enough so that the disks in the CBED pattern overlap. This is analogous to the elastic scattering case, where an interference of overlapping CBED disks is a necessary condition for atomic resolution STEM images [7].

An interesting corollary has been deduced on the basis of arguments of the coherence and symmetries present in the diffraction patterns: a nonzero EMCD should be detectable in the direction of the transmitted beam not only for vortex beams, but also for beams distorted by aberrations of suitable symmetry. For example, for tetragonal or cubic crystals one can utilize a nonzero antisymmetric fourfold astigmatism, i.e., $\mathrm{C}_{3,4 \mathrm{~b}}$ [6]. Similar symmetry considerations also provided a qualitative explanation, why electron vortex beams formed with spiral aperture do not lead to observable EMCD signal, despite that they can be rather easily focused to an atomic size, with full-width half-maximum sizes of $3 \AA$ or less [8].

With a clean vortex probe of sufficiently small size it should be possible to characterize magnetic moments at column-by-column basis. An example is shown in Fig. 1 summarizing simulated EMCD spectrum images for antiferromagnet $\mathrm{LaMnAsO}$ with a checkerboard pattern of magnetic ordering [9]. Simulations show that with a vortex of diameter $1.5 \AA$ one should be able to resolve differences of the order of 3-4\% at the Mn- $L_{2,3}$ edges, providing an EMCD signal from an antiferromagnetic compound - a feature that is out of reach in X-ray based techniques. Notice the doughnut shaped spots with a minimum at the position of the Mn atomic columns, when the probe is a vortex beam. 
Experimental realization of atomic resolution EMCD has not been published yet, however the first measurements with aberrated probes show very promising results [9]. In the analysis of experimental data one faces a problem of low signal to noise ratio - individual spectra in atomic resolution spectrum images measured at energy losses of several hundreds of electron-Volts are rather noisy. In such case the extraction of EMCD signal will profit from advanced statistical data processing techniques, such as blind source separation methods, image registration and unwarping or machine learning approaches.

\section{References:}

[1] P. Schattschneider et al., Nature 441, 486 (2006).

[2] M. Uchida, and A. Tonomura, Nature 464, 737 (2010).

[3] J. Verbeeck, H. Tian, and P. Schattschneider, Nature 467, 301 (2010).

[4] B. McMorran et al., Science 331, 192 (2011).

[5] H. Lidbaum et al., Phys. Rev. Lett. 102, 037201 (2009).

[6] J. Rusz, J. C. Idrobo, and S. Bhowmick, Phys. Rev. Lett. 113, 145501 (2014).

[7] S. J. Pennycook, and P. D. Nellist, Scanning Transmission Electron Microscopy: Imaging and Analysis (Springer, New York, 2011), Chap. 2.

[8] D. Pohl et al., Ultramic. 150, 15 (2015).

[9] J. C. Idrobo et al., submitted.

[10] This research was supported by the Swedish Research Council and STINT (J.R., A.E, J.S., S.B), and by the Center for Nanophase Materials Sciences (CNMS), which is sponsored at Oak Ridge National Laboratory by the Scientific User Facilities Division, Office of Basic Energy Sciences, U.S. Department of Energy (J.C.I.).
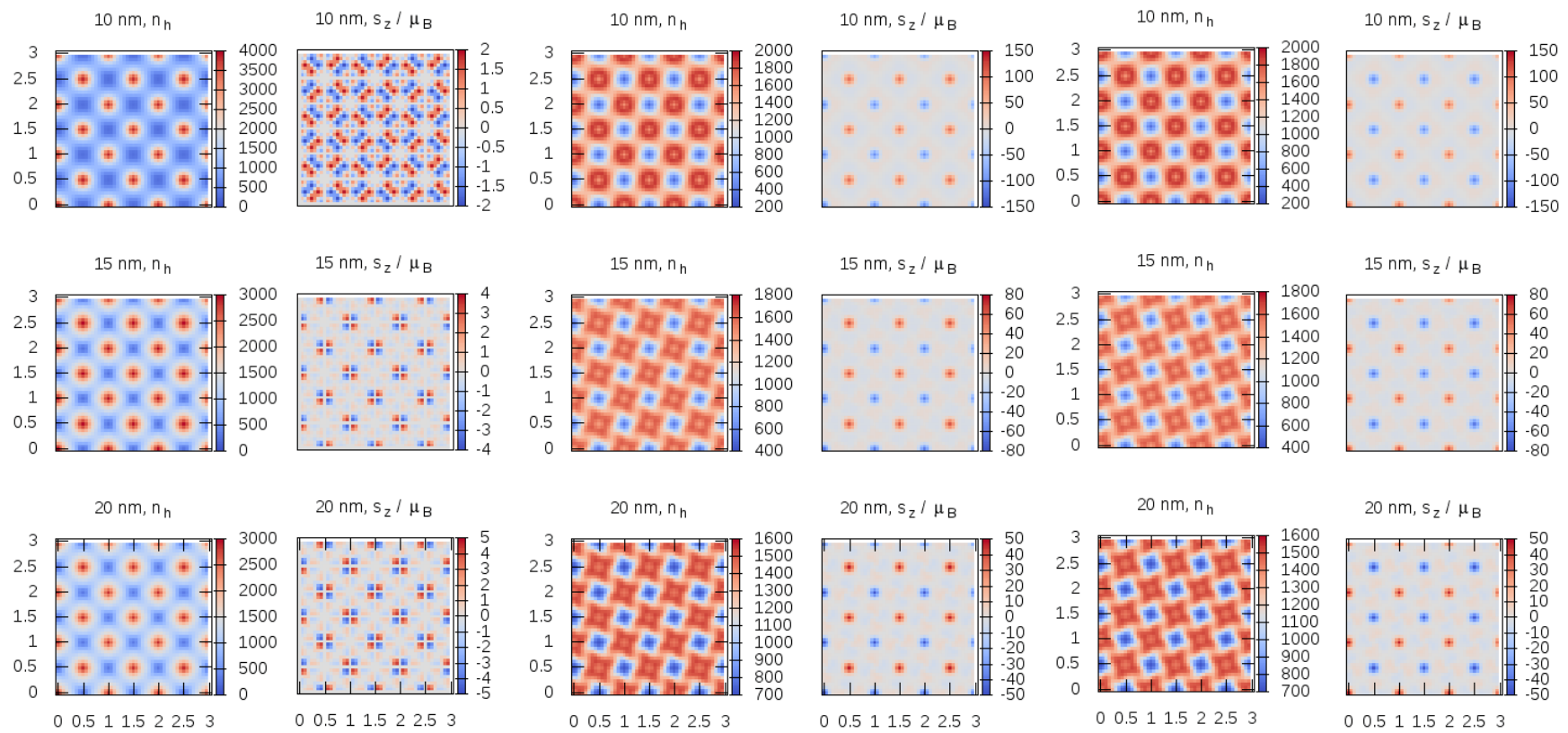

Figure 1. Simulated Mn- $L_{3}$ edge STEM spectrum images $\left(n_{h}\right)$ and pure magnetic signal component $\left(s_{z}\right)$ for an antiferromagnet LaMnAsO, assuming a probe with OAM=0 (left), +1 (middle) and -1(right) with corrected aberrations, $100 \mathrm{keV}$ acceleration voltage, 35mrad convergence angle and 45mrad collection angle. Sample thicknesses of 10nm, $15 \mathrm{~nm}$ or $20 \mathrm{~nm}$ have been considered. 